\title{
ВЛИЯНИЯ ВЫСОТ ЛОПАТОК РАБОЧЕГО КОЛЕСА ЦЕНТРОСТРЕМИТЕЛЬНОЙ ТУРБИНЫ НА ГАЗОДИНАМИКУ ТЕЧЕНИЯ
}

\author{
Пассар Андрей Владимирович, \\ passar_av@mail.ru \\ Вычислительный центр ДВО РАН, \\ Россия, 680000, г. Хабаровск, ул. Ким Ю Чена, 65
}

\begin{abstract}
Актуальность исследования определяется необходимостью создания эффективных газотурбинных двигателей, применяемых в отрасли нефтегазового комплекса.

Цель: при помощи модели пространственного течения газа в центростремительной турбине исследовать воздействие расчетного режима проектирования турбины на газодинамику течения в её рабочем колесе.

объект: центростремительная турбина энергоустановки малой мощности применяемого в отрасли нефтегазового комплекса. Методы. Для описания течения в турбине применяются уравнения пространственного потока. Для приведения этой системы уравнений к системе уравнений по двум координатам применяется метод прямых. Полученная система уравнений двумерного течения решается методом последовательных приближений.

Результаты. На основе метода оптимального проектирования спроектированы рабочие колеса центростремительной турбины для различных расчетных давлений газа на входе. Результаты проектирования показали, что с увеличением расчетного давления газа на входе в турбину уменьшаются площади входного и выходного сечений проточной части. На базе одномерной модели течения в турбинах показано влияние высот лопатки рабочего колеса на КПД и мощность турбины. В результате проведения одномерного расчета можно констатировать, что с увеличением высот лопатки рабочего колеса взрастает КПД турбины, а эффективная мощность падает. Впервые для центростремительной турбины показано воздействие расчетного давления газа на входе в турбину, на газодинамику течения в её рабочем колесе. В результате проведенных исследований установлено, что с уменьшением расчетного давления газа на входе в турбину возрастает область отрыва течения в рабочем колесе. Линия области отрыва течения определена при условии равенства нулю меридианной проекции относительной скорости. Представлены экспериментальный и расчетный профиль скоростей за проточной частью турбины. При сопоставлении расчетного профиля скоростей с экспериментальным следует отметить, что применяемая модель расчета двумерного течения в турбине позволяет с хорошей точностью рассчитать газодинамику течения в ней.
\end{abstract}

\section{Ключевые слова:}

Площадь проходного сечения, центростремительная турбина, КПД турбины, микротурбина, поле скоростей, линия тока, высота лопатки, энергоустановка.

\section{Введение}

В настоящее время в отрасли нефтегазового комплекса используются энергоустановки производства компании «Дрессер-Рэнд». В отдельную группу энергетических установок следует отнести микротурбины малой электрической мощностью [1-3], производимые фирмой «Кэпстоун», применяемые в отрасли нефтегазового комплекса. Топливом для них может служить природный газ, а также попутный нефтяной газ. Применение современных микротурбин в нефтяной промышленности позволяет утилизировать попутный нефтяной газ с низким содержанием сероводорода.

Одним из основных узлов микротурбинного двигателя является одноступенчатая центростремительная турбина. Центростремительная конструкция энергоустановок обеспечивает надежность, компактность и высокую производительность.

Работа, направленная на повышение эффективности центростремительной турбины энергоустановки, является актуальной. В [4-6] представлена методика определения расчетного режима проектирования турбины. Однако статьи, направленной на исследование влияния расчетного режима на газодинамику течения в проточной части турбины, до настоящего времени не было.
Цель настоящей работы: при помощи модели двумерного течения невязкого сжимаемого газа в проточной части центростремительной турбины исследовать воздействие расчетного режима проектирования турбины на газодинамику течения в её проточной части.

Для достижения этой цели поставлены и решены следующие задачи:

1. Используя метод оптимального проектирования, выполнен проектный расчет центростремительной турбины для различных расчетных режимов и построены проточные части.

2. Используя одномерную модель течения в турбине, выполнен расчет характеристик турбин и проведена его экспериментальная проверка.

3. Выполнен расчет двумерного течения невязкого сжимаемого газа в проточных частях турбин, и проведена его экспериментальная проверка.

4. На основе выполненного расчета выбран оптимальный расчетный режим и соответствующие ему проточные части.

\section{Математическая модель исследования}

На сегодняшний день на рынке программного обеспечения имеется множество коммерческих пакетов вычислительной гидродинамики [7-14], позволяющих производить расчет пространственно- 
го течения в турбинах. Использование этих пакетов заключается в решении численными методами двумерных и трехмерных уравнений, описывающих течение в турбомашинах. Эти пакеты включают разнообразные модели турбулентности. В работе [15] рассмотрено использование пакета ANSYS $C F X$ для решения уравнений двумерного и трехмерного течения в малорасходной осевой турбине. При этом время расчета двумерного течения в турбине занимает в среднем около 2 ч. Расчет производился на компьютере Core $i$, с тактовой частотой 2,7 ГГц и оперативной памятью 16 Гб.

В работах [16-18] рассмотрен расчет двумерного вихревого течения невязкого сжимаемого газа в полости центростремительной турбины с радиусом крылатки 70 мм. При этом время расчета на персональной машине занимает в среднем около 0,32 c.

Таким образом, по затратам машинного времени применение математической модели двумерного вихревого течения невязкого сжимаемого газа для моделирования течения в турбине эффективнее полноценного $C F D$-моделирования.

Математическая модель двумерного течения газа в центростремительной турбине подробно описана в работах [16-18]. Для описания течения применяются следующие уравнения, записанные в системе координат, связанной с проточной частью: движения, неразрывности, первого закона термодинамики. Отличительная черта этой модели - это то, что в ней для описания течения в проточной части турбины применяются уравнения для невязкого газа, при этом потери энергии в полости турбины учитываются косвенно, через расчет энтропии. А энтропия в свою очередь рассчитывается через коэффициент потерь в рабочем колесе.

Для приведения системы уравнений пространственного течения к системе уравнений по двум координатам применяется метод прямых [19]. Принято предположение об осевой симметрии потока [20, 21]. Частные производные заменяются конечными разностями $[22,23]$. Полученная система уравнений двумерного течения решается методом итераций $[24,25]$. Алгоритм решения рассматриваемой задачи подробно рассмотрен в работе [17].

Граничные условия.

1. Линии меридионального контура - линии тока.

2. Вход в проточную часть: течение вихревое, все частные производные по длине линии тока равны нулю.

3. Выход из проточной части: течение вихревое, все частные производные по длине линии тока равны нулю.

\section{Проектирование проточной части}

Для проектирования проточной части задаются следующие параметры: расход газа, проходящего через проточную часть; частота вращения турбины; давление торможения перед турбиной.

Число оборотов определяется при расчете оптимальных параметров турбины [26].
Принятые параметры четырех расчетных режимов приведены в таблице.

таблица. Параметры расчетных режимов

Table. $\quad$ Parameters of the calculated modes

\begin{tabular}{|l|c|c|c|c|}
\hline \multicolumn{1}{|c|}{$\begin{array}{l}\text { Параметры } \\
\text { Characteristis }\end{array}$} & \multicolumn{4}{|c|}{ Режим/Mode } \\
\cline { 2 - 5 } & 1 & 2 & 3 & 4 \\
\hline $\begin{array}{l}\text { Paсход } G_{t}, \text { кг } / \mathrm{c} \\
\text { Consumption } G_{t}, \mathrm{~kg} / \mathrm{s}\end{array}$ & 0,45 & 0,45 & 0,45 & 0,45 \\
\hline $\begin{array}{l}\text { Давление заторможенного потока на } \\
\text { входе в ступень } \\
\text { Pressure of the inhibited flow at the } \\
\text { stage inlet } p_{0}^{*}, \mathrm{MPa}\end{array}$ & 0,18 & 0,20 & 0,22 & 0,24 \\
\hline $\begin{array}{l}\text { Cтепень понижения давления } \\
\text { Pressure reduction degreе } \pi_{t . r}=p_{0}{ }^{*} / p_{2}\end{array}$ & 1,7 & 1,89 & 2 & 2,26 \\
\hline $\begin{array}{l}\text { Чacтота вращения } n_{\text {тк }}, \text { мин }^{-1} \\
\text { Rotational speed } n_{t k}, \text { min }^{-1}\end{array}$ & 35860 & 39880 & 43660 & 46860 \\
\hline
\end{tabular}

Используя метод оптимального проектирования [26], выполним проектный расчет проточных частей для определенных ранее расчетных режимов (таблица). Результаты проектирования представлены на (рис. 1). Угол закрутки лопаток направляющего аппарата $\alpha_{1}=18^{\circ}$. Угол закрутки лопаток рабочего колеса на среднем радиусе $\beta_{2}=36^{\circ}$. Лопатки рабочего колеса для обеспечения прочности и жесткости имеют радиальное направление в сечениях плоскостями, перпендикулярными оси колеса, а также малую толщину на периферии при значительной толщине у корня. Конфигурация линий меридионального контура представляет собой эллиптические кривые [17]. Также на рис. 1 для сравнения приведена проточная часть рабочего колеса штатной турбины турбокомпрессора TKP-14.

На рис. 1 турбина № 1 спроектирована на режим № 1 , турбины № 2 - на режим № 2 , турбина № 3 - на режим № 3, турбина № 4 - на режим № 4. Далее будем их называть опытными турбинами. Количество лопаток рабочего колеса для турбины № $1 z_{\text {рк }}=12$, для турбины № $2 z_{\text {рк }}=12$, для турбины № $3 z_{\mathrm{pk}}=13$, для турбины № $4 z_{\mathrm{pk}}=13$. Степень радиальности для всех рабочих колес $\mu=R_{\text {av }} / R_{1}=0,52$.

Как показали результаты проектирования, с увеличением расчетного давления газа на входе в турбину уменьшаются площади проходного сечения на входе и выходе из рабочего колеса (рис. 1), а также увеличивается оптимальное число оборотов турбины, определенное с помощью метода оптимального проектирования (таблица).

\section{Определение КПД турбин}

Для оценки проточных частей турбин, используя одномерную модель, выполнен расчет следующих зависимостей: КПД турбины от коэффициента напора $\eta_{\mathrm{r}}=f\left(\bar{H}_{\mathrm{r}}\right)$ (рис. $\left.2, a\right)$ и мощность турбины в функции коэффициента напора $N_{\mathrm{r}}=f\left(\bar{H}_{\mathrm{r}}\right)$ (рис. 2, б). Одномерная модель для расчета турбины основана на использовании уравнения энергии, расхода и моментов количества движения. Для определения 

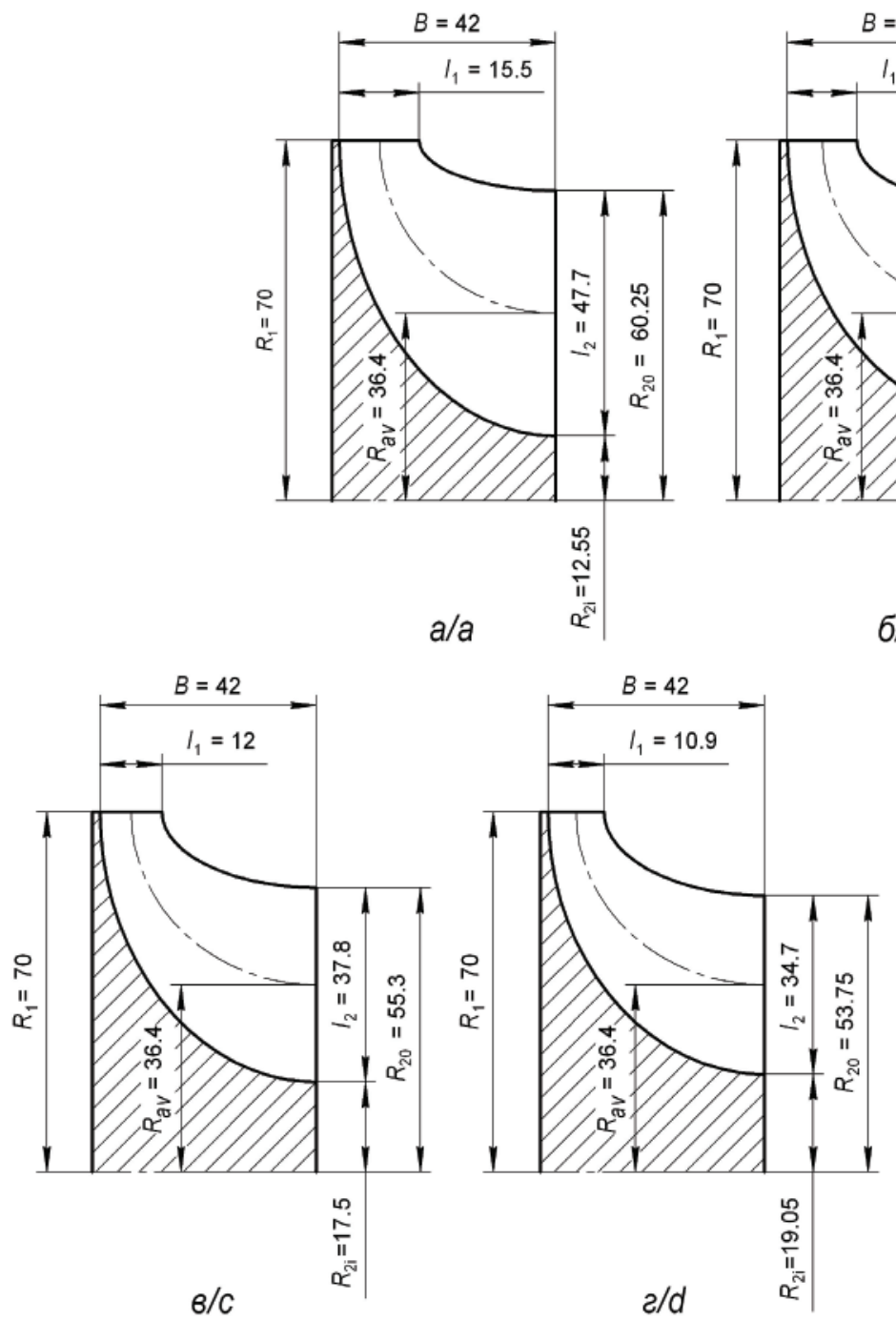
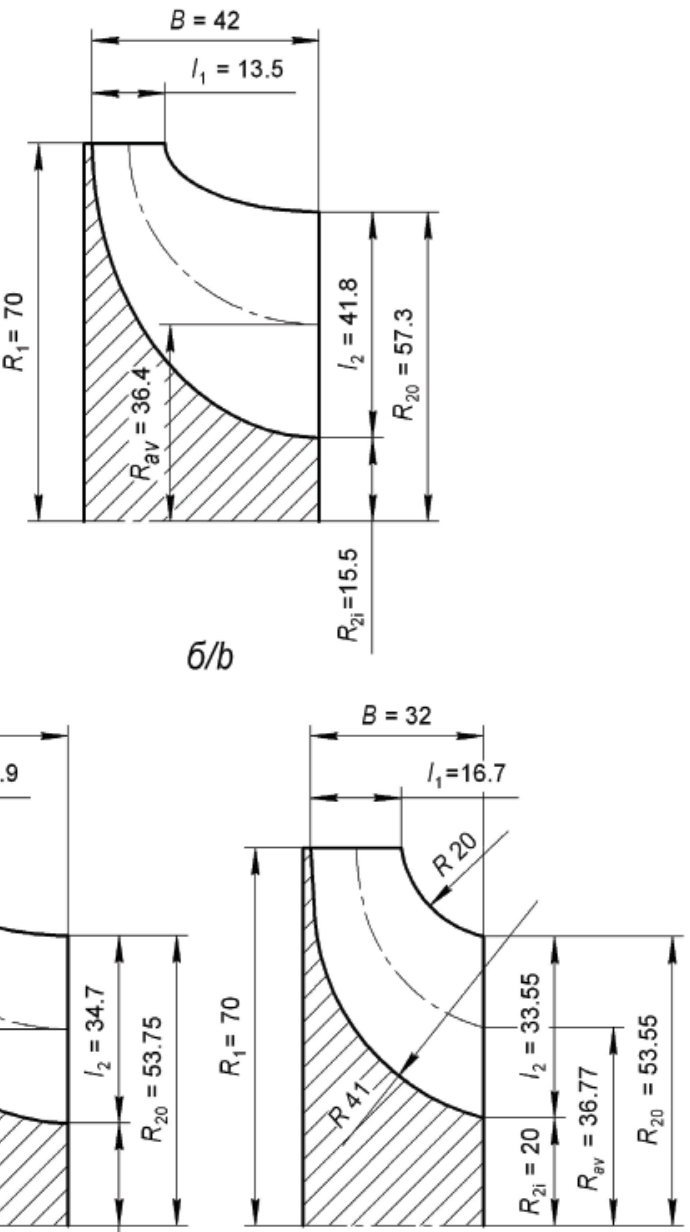

$\partial / e$

Рис. 1. Результаты оптимального проектирования: а) турбина № 1, режим 1; б) турбина № 2, режим 2; в) турбина № 3, режил 3; г) турбина № 4, режим 4; $)$ ) штатная турбина ТКР-14C-27

Fig. 1. Results of optimal design: a) turbine № 1, mode 1; b) turbine № 2, mode 2 ; C) turbine № 3, mode 3; d) turbine № 4, mode 4; d) regular turbine TKR-14S-27

потерь энергии в проточной части используются экспериментальные зависимости [27]. Коэффициент напора здесь принимается $\bar{H}_{\mathrm{T}}=2 h_{\text {ад }} / u_{1}^{2}, h_{\text {ад }}-$ текущее значение изоэнтропийного теплоперепада; $u_{1}$ - окружная скорость на входе в рабочее колесо. Из результатов расчета зависимостей $\eta_{\mathrm{T}}=f\left(\bar{H}_{\mathrm{T}}\right)$, $N_{\mathrm{T}}=f\left(\bar{H}_{\mathrm{r}}\right)$ следует:

1. На рассмотренном интервале изменения $\bar{H}_{\mathrm{r}}$ увеличение площади проходного сечения на входе и выходе из рабочего колеса приводит к увеличению эффективности турбины (рис. $2, a$ ).

2. На рассмотренном интервале изменения $\bar{H}_{\text {т }}$ увеличение площади проходного сечения на входе и выходе из рабочего колеса приводит к уменьшению эффективной мощности турбины (рис. 2, б).

3. На рассмотренном интервале изменения напора опытные турбины эффективнее штатной (рис. 2, $a$ ).
Проверка адекватности одномерной модели расчета турбины, выполненная путем сравнения расчетных зависимостей $\eta_{\mathrm{T}}=f\left(\bar{H}_{\mathrm{T}}\right), N_{\mathrm{T}}=f\left(\bar{H}_{\mathrm{T}}\right)$ для штатной турбины с экспериментальными данными, показала, что ошибка счета не превосходит величины $2 \%$ (рис. 2).

На основании одномерного расчета можно сделать следующий вывод: с уменьшениел площади проходного сечения на входе и выходе из рабочего колеса КПД турбины падает, а эффективная мощность растет за счет увеличения степени понижения давления и оптилальной частоты вращения турбины.

\section{Результаты расчетов}

Результаты одномерного расчета в точках максимума КПД на зависимостях $\eta_{\mathrm{T}}=f\left(\bar{H}_{\mathrm{T}}\right)$ (рис. $\left.2, a\right)$ использовались в качестве начального приближения для двумерного расчета. Для выполнения дву- 

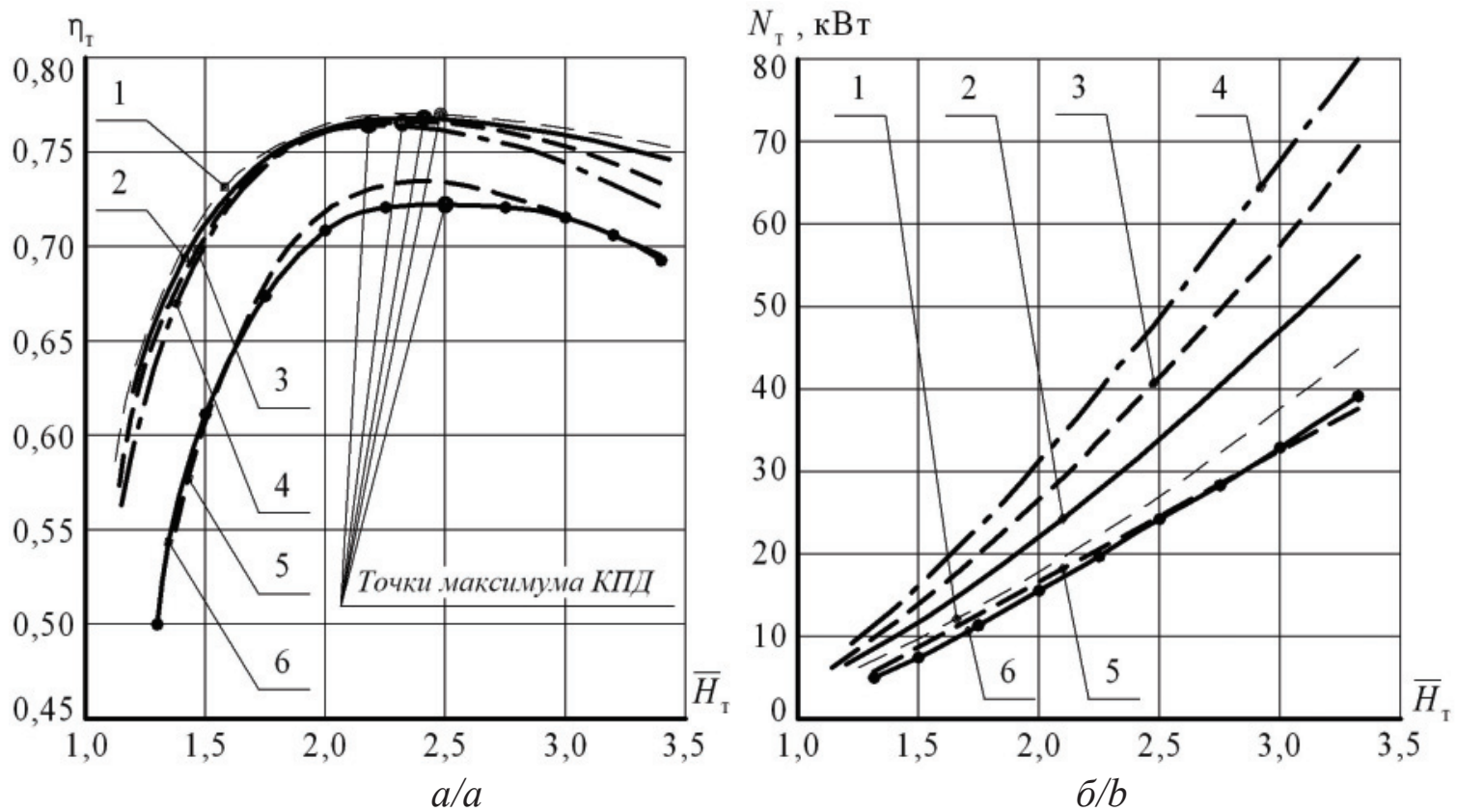

Pис. 2. Влияние расчетного режима на КПД и мощность: а) КПд турбины; б) эффективная мощность; 1 - турбина № 1; 2 - турбина № 2; 3 - турбина № 3; 4 - турбина № 4; 5 - расчетнал характеристика штатной турбины ТКР-14С-27; 6 - эксперилентальная характеристика турбины ТКР-14C-27

Fig. 2. Impact of the design mode on efficiency and power: a) turbine efficiency; b) effective power; 1 - turbine № 1 ; 2 - turbine № 2; 3 - turbine № 3; 4 - turbine № 4; 5 - design characteristics of the turbine TKR-14S-27;6 - experimental characteristics of the turbine TKR-14S-27

мерного расчета течения газа в проточной части центростремительной турбины составлена программа на языке программирования MATLAB [28].

Паралетры сетки. Для проведения расчета проточная часть разбивается на сетку. Для этого проводим семейство прямых $l$ к внешней линии меридионального контура (рис. 3). После этого наносим по принципу равных кольцевых площадей линии тока $s$ (на рис. 3 показаны тонкими линиями). Для обеспечения сходимости приближений существенно, чтобы $l$ мало отличалась от нормали к линии тока. Выполнение этого условия совместно с требованием $\Delta l \leq \Delta s$ практически обеспечивает сходимость последовательных приближений.

На рис. 3 показаны результаты моделирования динамики двумерного течения невязкого сжимаемого газа, проведенные в рабочих колесах центростремительных турбин, спроектированных на различные расчетные режимы. Как показано на рис. 3, поверхности тока последнего приближения (толстые линии) существенно отличаются от линий предварительного разбиения канала (тонкие линии).

Всего для необходимой сходимости (2\% по скорости) потребовалось выполнить от 12 до 15 приближений. Время, затраченное машиной на одно приближение, составило 0,016 с. В рассматриваемом примере коэффициент релаксации равен $\alpha=0,5$.
На рис. 3 показано, что с увеличением кривизны линий меридионального контура рабочего колеса линии тока приближенны к кожуху турбины. Преимущественно это выражено в турбине № 1 в зоне прямых $l_{5}, l_{6}$ и $l_{7}$. Слабее это выражено в турбинах № 2 и 3 в зоне прямых $l_{6}$ и $l_{7}$. В области этих прямых на внутреннем контуре меридионального профиля появляются отрицательные скорости. Это обстоятельство дает основание предполагать, что в рабочих колесах турбины образуется отрыв течения (возвратные течения). На рис. 3 область возвратных течений заштрихована. Граница области возвратных течений определена при условии равенства нулю меридиональной проекции скорости. При увеличении расчетного давления газа на входе в турбину линии тока несколько выравниваются. К примеру, в проточной части № 4 линии тока более ровные, чем в проточных частях № 1,2 и 3 .

Результаты исследований свидетельствуют о тол, что возрастание высот лопаток рабочего колеса на входе $l_{1}$ и выходе $l_{2}$ приводит $\kappa$ возрастанию КПД турбины (рис. 2), падению әффективной мощности турбины, а также в проточной части турбины увеличивается область возвратных течений (рис. 3 ).

Здесь же хотелось бы отметить, что возможности моделирования отрывных течений в центростремительных турбинах в рамках невязкого газа подробно рассмотрены в работе В.Т. Митрохина [26]. 

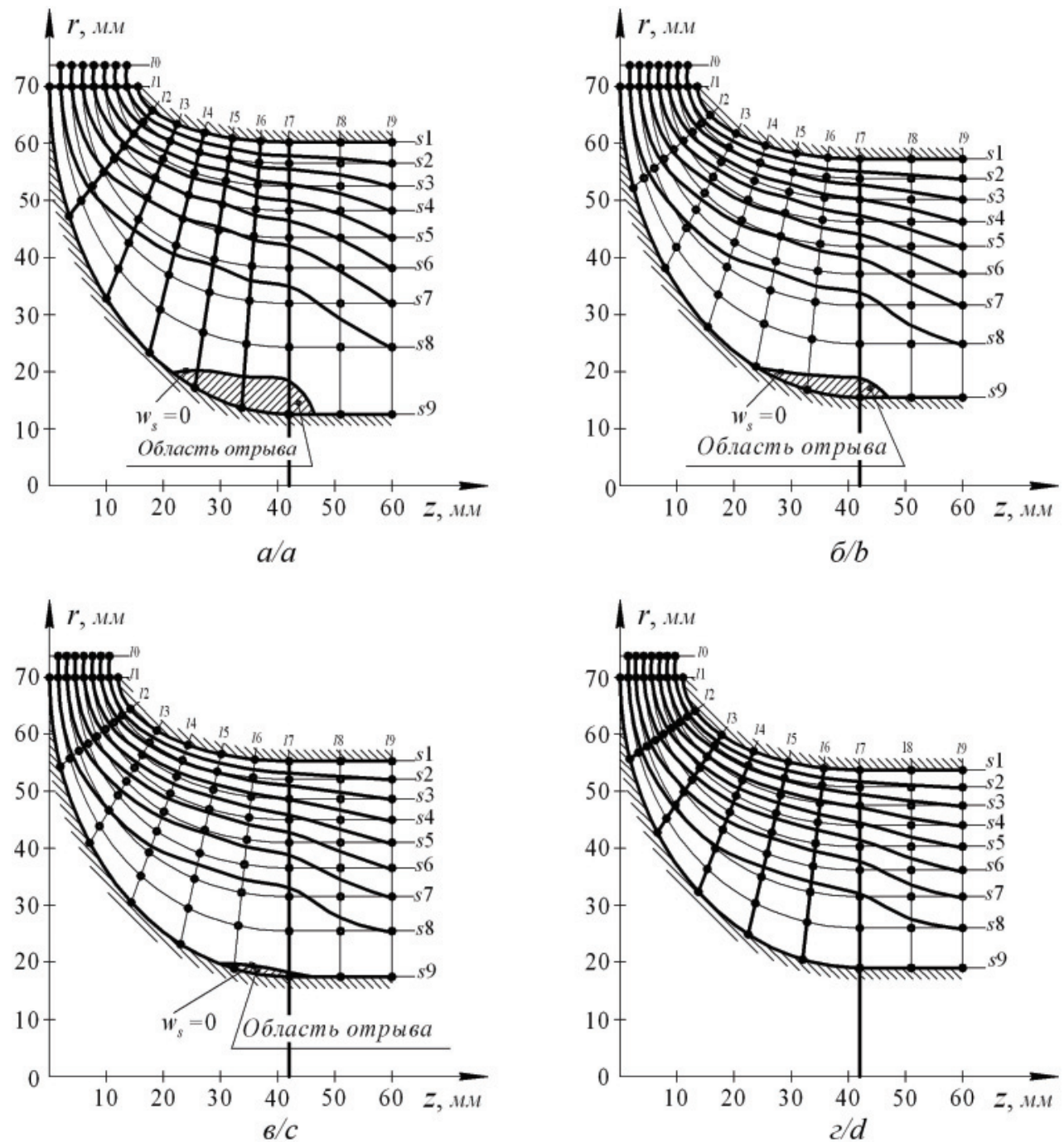

Рис. 3. Результаты расчета течения в полостях иентростремительных турбин: а) турбина № 1- режим № 1; б) турбина № 2 - режим № 2; в) турбина № 3 - режим № 3; г) турбина № 4 - режим № 4

Fig. 3. Results of flow calculation in the cavities of centripetal turbines: a) turbine № 1 - mode № 1; b) turbine № 2 - mode № 2; c) turbine № 3 - mode № $3 ; d$ ) turbine № 4 - mode № 4

\section{Экспериментальная проверка модели}

Для экспериментальной проверки математической модели двумерного вихревого течения невязкого сжимаемого газа в проточной части центростремительной турбины проведена продувка турбины турбокомпрессора TКР-14C-27 на специальном стенде. Стенд позволяет по измеряемым параметрам определять КПД турбины рис. 2, а также производить замер поля скорости на выходе из проточной части турбины. Описание стенда приведено в работах $[16,17]$. Для замеров параметров потока за турбиной, в районе выхода газа из рабочего колеса, на стенд установлен шаровой зонд с координатным устройством. Кроме замера полного давления зонд позволяет измерять угол выхода потока из турбины.

Для проверки адекватности был произведен расчет газовой динамики двумерного течения в проточной части штатной турбины TКР-14C-27 на режиме максимума КПД и режиме отрывного течения. Результаты расчета приведены на рис. 4. Экспериментальная проверка на режиме максимума КПД производилась следующим образом. Сравнивалось расчетное поле абсолютной скорости на выходе из рабочего колеса с экспериментально замеренным полем скорости относительно неподвижной системы координат. В решение задачи вносились поправки экспериментальными значениями углов $\alpha_{2}$, замеренными в сечениях $l_{8}$ и $l_{9}$ (рис. 4 ).

Для статистической обработки экспериментально замеренного поля скорости использовались возможности программы Mathcad [29, 30]. Для расчета доверительного интервала использовалось распределение Стьюдента.

Сопоставление рассчитанного профиля скорости с экспериментально замеренным показывает хорошее совпадение в средней и привтулочной областях течения (рис. 5). 

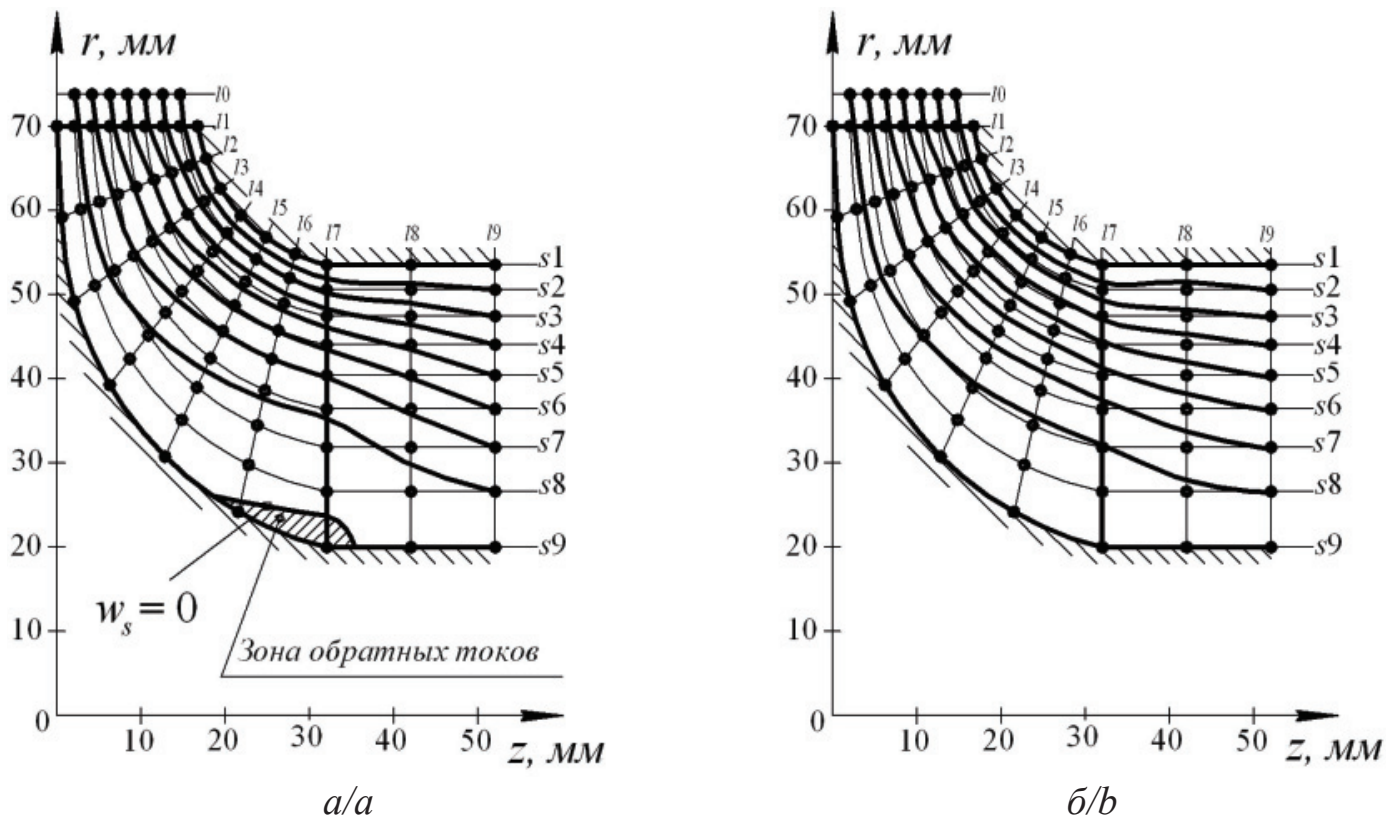

Pис. 4. Результаты расчета течения в полостях штатной турбины: а) режим отрывного течения; б) режим максимума КПд

Fig. 4. Results of calculation of the flow in the cavities of the regular turbine: a) separated flow mode; b) maximum efficiency mode

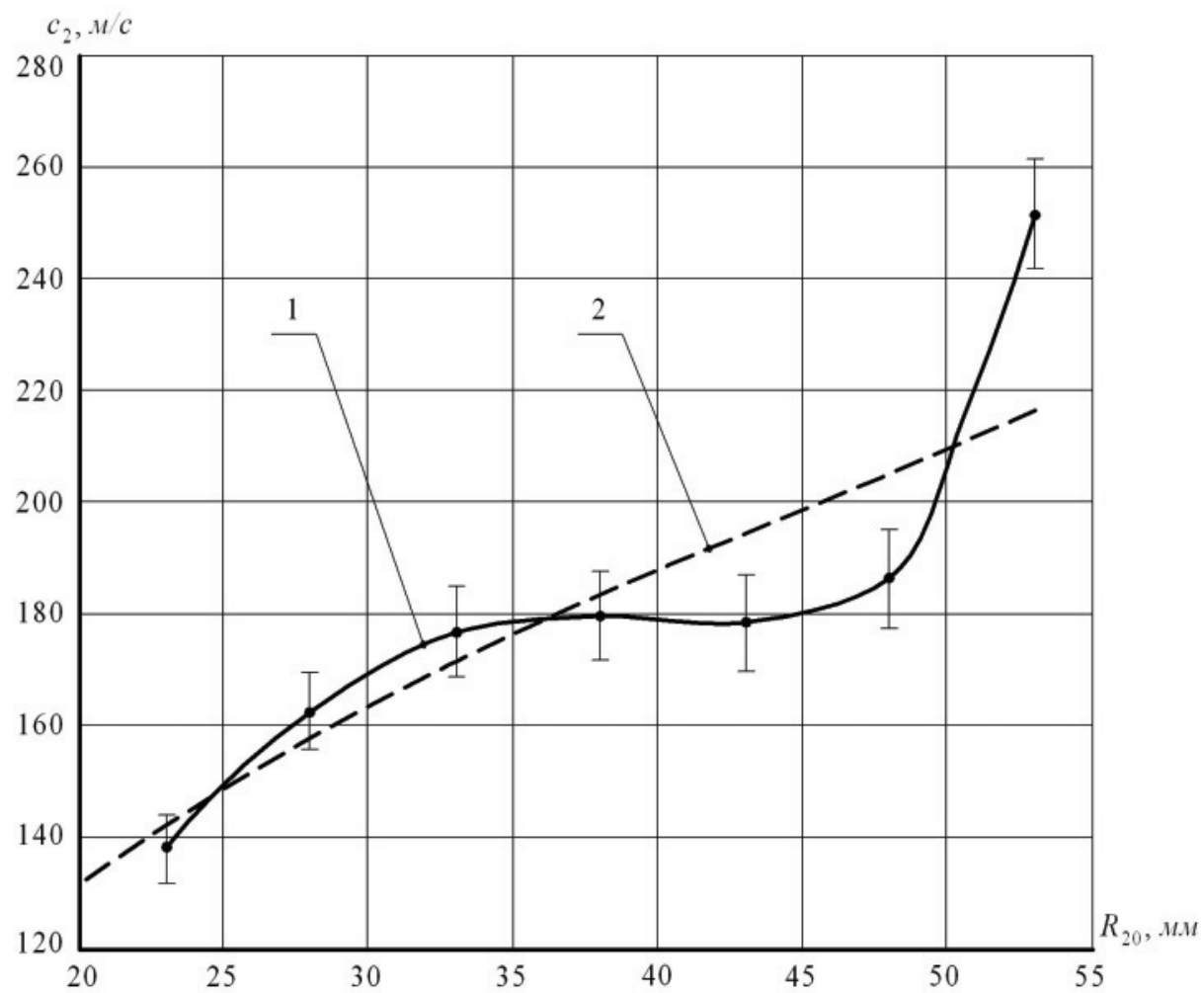

Pис. 5. Экспериментальный и расчетный профиль скоростей на выходе из турбины на режиме максимума КПД: 1 - экспериментальная кривая; 2 - расчетная кривая после 14 приближений с корректировкой экспериментальными значенияли углов $\alpha_{2}$

Fig. 5. Experimental and calculated velocity profile at the turbine outlet at the maximum efficiency mode: 1 - experimental curve; 2 - calculated curve after 14 approximations with correction of experimental angles $\alpha_{2}$

Метод расчета двумерного течения не учитывает влияния переднего осевого зазора, особенно в выходном сечении, вызывающего искажение структуры потока на периферии. Поэтому погрешность расчета в этой области несколько больше (9-14 \%), чем в остальной части выходного сечения (рис. 5).

В основном расчет течения на режиме максимума КПД можно считать удовлетворительным, по- 
скольку расхождения между вычисленными и экспериментальными значениями $c_{2}$ в средней и привтулочной областях не выходят за границы доверительного интервала (рис. 5).

Эксперилентальная проверка на режиле отрывного течения. Начало области отрыва течения по радиусу располагается в привтулочной области. Для определения области отрыва за рабочим колесом измерялись и сравнивались между собой давление торможения и статическое давление. Сложность при этом составляло определение угла выхода потока. Вблизи области отрыва течения поворот измерительного устройства в диапазоне от $-10^{\circ}$ до $+10^{\circ}$ не приводил к перемене давления торможения. Вследствие этого окончательное суждение о появлении области отрыва регистрировалось при равенстве давления торможения и статического давления.

Экспериментально измеренные давления по высоте выходного сечения проточной части приведены на рис. 6 . Как показано на рис. 6 , в прикорневой области появляется срыв потока. Протяженность зоны срыва течения вдоль выходного сечения рабочего колеса устанавливалась тогда, когда давление торможения было равно статическому давлению.

Сравнение расчетных (рис. $4, a$ ) и экспериментальные данных (рис. 6) на режиме отрыва течения позволяет сделать заключение: предположение, о том, что в турбине возникает отрыв потока, нашло экспериментальное подтверждение.

Результаты данного эксперимента можно распространить и на опытные турбины № 1,2 и 3 , в которых возникает зона возвратных течений, так как эти турбины и штатная турбина относятся к одному типоразмеру.

\section{Выводы}

Результаты проведенных исследований свидетельствуют о следующем:

1. Проверка адекватности одномерной модели расчета турбины, выполненная путем сравнения расчетных зависимостей $\eta_{\mathrm{T}}=f\left(\bar{H}_{\mathrm{T}}\right), N_{\mathrm{T}}=f\left(\bar{H}_{\mathrm{T}}\right)$ штатной турбины с экспериментальными данными, показала, что погрешность расчета не превышает величины $2 \%$.

2. Во всем интервале изменения напора опытные турбины эффективнее штатной.

3. Понижение расчетного давления перед турбиной ведет к возрастанию высот лопатки рабочего колеса на входе $l_{1}$ и выходе $l_{2}$, возрастанию КПД турбины, падению эффективной мощности, а также в проточной части турбины возрастает область возвратных течений. На основании этого при проектировании центростремительной турбины рекомендуем принимать значение полного давления газа на входе в турбину $p_{0}^{*}=0,24$ МПа. Это позволит избежать области возвратных течений.

4. При увеличении расчетного давления газа на входе в турбину линии тока несколько выравниваются. К примеру, в рабочем колесе турбины № 4 линии тока более гладкие, чем в рабочих колесах турбин № 1,2 и 3.

5. Сопоставление рассчитанного профиля скорости с экспериментально замеренным не режиме

$p_{2}, p_{2}{ }^{*}, M \Pi a$

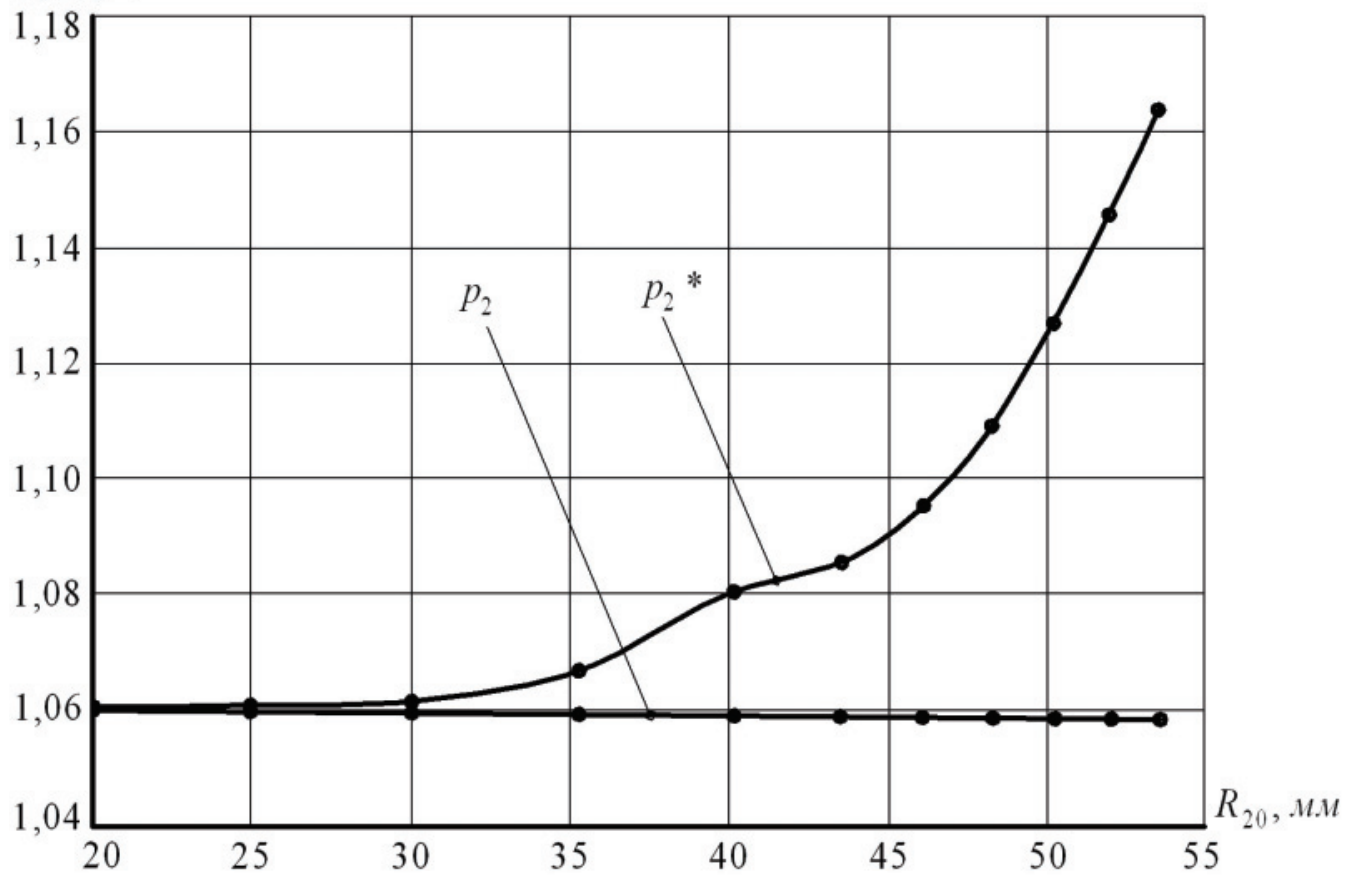

Puc. 6. Изленение давления торложения и статического давления по высоте выходного сечения

Fig. 6. Change of braking pressure and static pressure in the height of the output section 
максимума КПД показывает хорошее совпадение в центральной и прикорневой областях течения. Метод расчета двумерного течения не учитывает влияния переднего осевого зазора, особенно в выходном сечении, вызывающего искажение структуры потока на периферии. Поэтому погрешность расчета в этой области нес-

\section{СПИСОК ЛИТЕРАТУРЫ}

1. Feng Z.P., Deng Q.H., Li J. Aerothermodynamic design and numerical simulation of radial inflow turbine impeller for a $100 \mathrm{~kW}$ microturbine // Turbo Expo 2005: Power for land, sea, and air. Nevada, 2005. - V. 1. - P. 873-880.

2. Experimental validation of an integrated optimization design of radial turbine for micro gas turbines / L. Fu, Z.P. Feng, G.J. Li, Q.H. Deng, Y. Shi, T.Y. Gao // Journal of Zhejiang university science. - March 2015. - V. 16. - Iss. 3. - P. 241-249.

3. Murugan D.M., Tabakoff W., Hamed A. Three-dimensional flow field measurements using LDV in the exit region of a radial in flow turbine // Experiments in fluids. - May 1996. - V. 21. Iss. 1. - P. 1-10

4. Байков Б.П. Особенности расчета турбины, работающей на газах переменного давления // Труды Центрального научно-исследовательского дизельного института. - 1955. - Вып. 28. C. $68-87$.

5. Иванов П.В. Расчет радиальной импульсной турбины с учетом перетекания и упрощенный расчет // Труды Центрального научно-исследовательского дизельного института. - 1960. Вып. 39. - С. 66-73.

6. Пассар А.В., Лашко В.А. Выбор расчетного режима и оценка эффективности турбины в нестационарном потоке // Справочник. Инженерный журнал с приложением. - 2011. № 2 (167). - C. 17-22.

7. Bayomi N.N., Abd EI-Maksoud R.M. Two operating modes for turbocharger system // Energy Conversion and Management. 2012. - № 58. - P. 59-65.

8. Binder N., Garcia B.J., Carbonneau X. Dynamic response in transient operation of a variable geometry turbine stage: influence of the aerodynamic performance // International Journal of Rotating Machinery. - 2013. - Article ID 735321. - P. 1-11.

9. Ghasemi S., Shirani E., Hajilouy-Benisi A. Performance prediction of twin-entry turbocharger turbines // Turbo Expo 2002: ASME. - Amsterdam, 2002. - V. 1. - P. 1087-1095.

10. Rotor-blades profile influence on a gas-turbine's compressor effectiveness / B.T. Lebele-Alawa, H.I. Hart, S.O.T. Ogaji, S.D. Probert // Applied Energy. - 2008. - № 85. - P. 494-505.

11. Kou H.J., Lin J.S., Zhang J.H. Numerical study on vibration stress of rotating fan blade under aerodynamic load at critical speed // Journal of Aerospace Engineering. - 2015. - № 230 (6). P. 1044-1058.

12. Newton P., Martinez-Botas R., Seiler M. A three-dimensional computational study of pulsating flow inside a double entry turbine // Journal of Turbomachinery. - 2014. - V. 137. - P. 1-10.

13. Comparison between the steady performance of double-entry and twin-entry turbocharger turbines / A. Romagnoli, C.D. Copeland, R. Martinez-Botas, M. Seiler, S. Rajoo, A. Costall // Journal of Turbomachinery. - 2013. - V. 135. - P. 1-11.

14. Stolarski T., Nakasone Y., Yoshimoto S. Engineering analysis with ANSYS software. - Amsterdam: Elsevier, Academic Press, 2010. $-480 \mathrm{p}$. колько больше $(9-14 \%)$, чем в остальной части выходного сечения. В основном расчет течения на режиме максимума КПД можно считать удовлетворительным, поскольку расхождения между расчетными и экспериментальными значениями $c_{2}$ в средней и привтулочной зонах не выходят за границы доверительного интервала.

15. Епифанов А.А. Численное моделирование трехмерного течения в решетках и ступенях малорасходных турбин ЛПИ: автореф. дис. ... канд. техн. наук. - СПб., 2012. - 14 с.

16. Пассар А.В., Тимошенко Д.В. Исследование влияния степени радиальности на структуру потока в проточной части радиально-осевой турбины газотурбинной установки // Известия Томского политехнического университета. Инжиниринг георесурсов. - 2017. - Т. 328. - № 5. - С. 24-38.

17. Пассар А.В. Влияние формы меридионального контура рабочего колеса на параметры потока газа в радиально-осевой турбине газотурбинной установки // Известия Томского политехнического университета. Инжиниринг георесурсов. - 2017. T. 328. - № 9. - C. 33-48.

18. Пассар А.В. Влияние ширины рабочего колеса на эффективность радиально-осевой турбины // Машиностроение и инженерное образование. - 2017. - № 4 (53). - С. 61-73.

19. Зенков А.В. Численные методы. - М.: Изд-во «Юрайт», 2017. $-124 \mathrm{c}$

20. Wu Chung-Hua. A general theory of three-dimensional flow in subsonic and supersonic turbomachines of axial, radial and mixed-flow types // Transaction of the American Society of Mechanical Engineers. - 1952. - V. 74. - № 8. - P. 1363-1380.

21. Hawthorne W., Novak R. The aerodynamics of turbo-machinery // Annual review of fluid mechanics. - 1969. - V. 1. - № 4. - P. 341-366.

22. Pulliam T.H., Steger J.L. Implicit finite-difference simulation of three-dimensional compressible flow // American Institute of Aeronautics and Astronautics Journal. - 1980. - V. 18. - № 2. P. 159-167.

23. Chakravarthy S.R. Euler equation - implicit schemes and boundary conditions // American Institute of Aeronautics and Astronautics Journal. - 1983. - V. 21. - № 5. - P. 699-706.

24. Зализняк В.Е. Численные методы. Основы научных вычислений. - М.: Изд-во «Юрайт», 2016. - 356 с.

25. Численные методы / У.Г. Пирумов, В.Ю. Гидаспов, И.Э. Иванов, Д.Л. Ревизников, В.Ю. Стрельцов, В.Ф. Формалев. - М.: Изд-во «Юрайт», 2016. - 422 с.

26. Митрохин В.Т. Выбор параметров и расчет центростремительной турбины на стационарных и переходных режимах. - М.: Машиностроение, 1974. - 228 с.

27. Лашко В.А., Пассар А.В. Расчет коэффициента потерь кинетической энергии в проточной части как одна из проблем реализации комплексного подхода // Вестник Тихоокеанского государственного университета. - 2011. - № 1 (20). - С. 79-90.

28. Ревинская 0.Г. Основы программирования в MATLAB. - СПб.: Изд-во «БХВ-Петербург», 2016. - 208 с.

29. Воскобойников Ю.Е., Задорожный А.Ф. Основы вычислений и программирования в пакете Mathcad PRIME. - СПб.: Изд-во «Лань», 2016. -224 c.

30. Далингер В.А., Симонженков С.Д., Галюкшов Б.С. Теория вероятностей и математическая статистика с применением Mathcad. - М.: Изд-во «Юрайт», 2017. - 422 с.

Поступила 28.02.2019 2.

\section{Информация об авторах}

Пассар A.B., кандидат технических наук, старший научный сотрудник лаборатории «Численные методы математической физики» Вычислительного центра ДВО РАН. 


\title{
INFLUENCE OF BLADE HEIGHT IN CENTRIPETAL TURBINE IMPELLER ON FLOW GAS DYNAMICS
}

\author{
Andrey V. Passar, \\ passar_av@mail.ru \\ Computer center of Far East branch of the Russian Academy of Science, \\ 65, Kim U Chen street, Khabarovsk, 680000, Russia.
}

The relevance of the research is determined by the need to create effective gas turbine engines used in the oil and gas industry. The aim of the research is to investigate the impact of the turbine design mode on flow gas dynamics in its impeller using the model of spatial gas flow in a centripetal turbine.

Object: centripetal turbine of low power energy unit applied in the oil and gas industry.

Methods. Spatial flow equations are used to describe the flow in the turbine. To bring this system of equations to the system of equations in two coordinates, the method of lines is used. The obtained system of two-dimensional flow equations is solved by the method of successive approximations.

Result. Based on the optimal design method, the impellers of the centripetal turbine are designed for different design pressures of gas at the inlet. The design results showed that, with the increase of the calculated gas pressure at the turbine inlet, the area of the passage section at the inlet and outlet of the impeller decreases. On the basis of one-dimensional model of the flow in turbines the influence of the height of impeller blades on the turbine efficiency and power is shown. As a result of the research, it can be stated that with the increase in the height of the impeller blade, the efficiency of the turbine grows, and the effective power decreases. The effect of the calculated gas pressure at the turbine inlet on the gas dynamics of the flow in its impeller is shown for a centripetal turbine. As a result of the studies it was found that with a decrease in the calculated gas pressure at the turbine inlet, the region of the flow separation in the impeller increases. The line of the flow separation region is determined under the condition of zero Meridian projection of the relative velocity. The experimental and calculated velocity profile at the turbine outlet is presented. Comparison of the calculated velocity profile with the experimental one allows us to conclude that the applied model for calculating the two-dimensional flow in the turbine make it possible to calculate the gas dynamics of the flow in it with high accuracy.

Key word:

Flow section area, centripetal turbine, turbine efficiency, microturbine, velocity field, current line, blade height, power plant.

\section{REFERENCES}

1. Feng Z.P., Deng Q.H., Li J. Aerothermodynamic design and numerical simulation of radial inflow turbine impeller for a $100 \mathrm{~kW}$ microturbine. Turbo Expo 2005: Power for land, sea, and air. Nevada, 2005. Vol. 1, pp. 873-880.

2. Fu L., Feng Z.P., Li G.J., Deng Q.H., Shi Y., Gao T.Y. Experimental validation of an integrated optimization design of radial turbine for micro gas turbines. Journal of Zhejiang university science, March 2015, vol. 16, Iss. 3, pp. 241-249.

3. Murugan D.M., Tabakoff W., Hamed A. Three-dimensional flow field measurements using LDV in the exit region of a radial inflow turbine. Experiments in fluids, May 1996, vol. 21, Iss. 1, pp. 1-10.

4. Baykov B.P. Osobennosti rascheta turbiny, rabotayushchey na gazakh peremennogo davleniya [Features of calculation of the turbine operating on gases of variable pressure]. Proceedings of the Central research diesel Institute, 1955, vol. 28, pp. 68-87.

5. Ivanov P.V. Raschet radialnoy impulsnoy turbiny s uchetom peretekaniya i uproshchenny raschet [Calculation of radial impulse turbine taking into account overflow and simplified calculation]. Proceedings of the Central research diesel Institute, 1960, vol. 39, pp. 66-73.

6. Passar A.V., Lashko V.A. Selection of the design mode and evaluation of the turbine efficiency in a non-stationary flow. Directory. Engineering journal with Appendix, 2011, no. 2 (167), pp. 17-22. In Rus.

7. Bayomi N.N., Abd EI-Maksoud R.M. Two operating modes for turbocharger system. Energy Conversion and Management, 2012, no. 58. pp. 59-65.

8. Binder N., Garcia B.J., Carbonneau X. Dynamic response in transient operation of a variable geometry turbine stage: Influence of the aerodynamic performance. International Journal of Rotating Machinery, 2013. Article ID 735321, pp. 1-11.

9. Ghasemi S., Shirani E., Hajilouy-Benisi A. Performance prediction of twin-entry turbocharger turbines. Turbo Expo 2002: Ame- rican Society of Mechanical Engineers. Amsterdam, 2002. Vol. 1, pp. 1087-1095.

10. Lebele-Alawa B.T., Hart H.I., Ogaji S.0.T., Probert S.D. Rotorblades profile influence on a gas-turbine's compressor effectiveness. Applied Energy, 2008, no. 85, pp. 494-505.

11. Kou H.J., Lin J.S., Zhang J.H. Numerical study on vibration stress of rotating fan blade under aerodynamic load at critical speed. Journal of Aerospace Engineering, 2015, no. 230 (6), pp. 1044-1058.

12. Newton P., Martinez-Botas R., Seiler M. A three-dimensional computational study of pulsating flow inside a double entry turbine. Journal of Turbomachinery, 2014, vol. 137, pp. 1-10.

13. Romagnoli A., Copeland C.D., Martinez-Botas R., Seiler M., Raj00 S., Costall A. Comparison between the steady performance of double-entry and twin-entry turbocharger turbines. Journal of Turbomachinery, 2013, vol. 135, pp. 1-11.

14. Stolarski T., Nakasone Y., Yoshimoto S. Engineering analysis with ANSYS software. Amsterdam, Elsevier, Academic Press, 2010. $480 \mathrm{p}$.

15. Epifanov A.A. Chislennoe modelirovanie trekhmernogo techeniya $v$ reshetkakh $i$ stupenyakh maloraskhodnykh turbin LPI. Avtoreferat Dis. Kand. nauk [Numerical simulation of three-dimensional flow in lattices and stages of low-flow turbines of LPI. Cand. Diss. Abstract]. St-Petersburg, 2012. 14 p.

16. Passar A.V., Timoshenko D.V. Study of the influence of the degree of radiality on the flow structure in the flow part of the radial-axial turbine of a gas turbine installation. Bulletin of the Tomsk Polytechnic University. Geo Assets Engineering, 2017, vol. 328 , no. 5, pp. 24-38. In Rus.

17. Passar A.V. The influence of the shape of the meridional contour of the impeller on the parameters of gas flow in radial-axial turbine gas turbine. Bulletin of the Tomsk Polytechnic University. Geo Assets Engineering, 2017, vol. 328, no. 9, pp. 33-48. In Rus.

18. Passar A.V. The effect of the width of the impeller on the efficiency of the radial-axial turbine. Mechanical industry and engineering education, 2017, no. 4 (53), pp. 61-73. In Rus. 
19. Wu Chung-Hua. A general theory of three-dimensional flow in subsonic and supersonic turbomachines of axial, radial and mixed-flow types. Transaction of the American Society of Mechanical Engineers, 1952, vol. 74, no. 8, pp. 1363-1380.

20. Hawthorne W., Novak R. The aerodynamics of turbo-machinery. Annual review of fluid mechanics, 1969, vol. 1, no. 4, pp. 341-366.

21. Zenkov A.V. Chislennye metody [Numerical method]. Moscow, Yurayt Publ., 2017. 124 p.

22. Pulliam T.H., Steger J.L. Implicit finite-difference simulation of three-dimensional compressible flow. American Institute of Aeronautics and Astronautics Journal, 1980, vol. 18, no. 2, pp. $159-167$.

23. Chakravarthy S.R. Euler equation - implicit schemes and boundary conditions. American Institute of Aeronautics and Astronautics Journal, 1983, vol. 21, no. 5, pp. 699-706.

24. Zaliznyak V.E. Chislennye metody. Osnovy nauchnykh vychisleniy [Numerical methods. Fundamentals of scientific computing]. Moscow, Yurayt Publ., 2016. 356 p.

25. Pirumov U.G., Gidaspov V.Yu., Ivanov I.E., Reviznikov D.L., Streltsov V.Yu., Formalev V.F. Chislennye metody [Numerical methods]. Moscow, Yurayt Publ., 2016. 422 p.
26. Chumakov Yu.A. Teoriya i raschet transportnykh gazoturbinnykh dvigateley [Theory and calculation of transport gas turbine engines]. Moscow, INFRA-M; Forum Publ., 2012. 448 p.

27. Lashko V.A., Passar A.V. Calculation of kinetic energy losses coefficient in the flow range as one of the challenges of implementing an integrated approach. Bulletin of the Pacific national University, 2011, no. 1 (20), pp. 79-90. In Rus.

28. Revinskaya 0.G. Osnovy programmirovaniya $v$ MATLAB [The basics of programming in MATLAB]. St-Petersburg, BHV-Peterburg Publ., 2016. 208 p.

29. Voskoboynikov Yu.E., Zadorozhnyy A.F. Osnovy vychisleniy $i$ programmirovaniya $v$ pakete Mathcad PRIME [Fundamentals of computing and programming in Mathcad PRIME]. St-Petersburg, Lan Publ., 2016. 224 p.

30. Dalinger V.A., Simonzhenkov S.D., Galyukshov B.S. Teoriya veroyatnostey $i$ matematicheskaya statistika s primeneniem Mathcad [Probability theory and mathematical statistics using Mathcad]. Moscow, Yurayt Publ., 2017. 422 p.

Received: 28 February 2019.

\section{Information about the authors}

Andrey V. Passar, Cand. Sc., senior researcher, Computer center of Far East branch of the Russian Academy of Science. 